\title{
POEMS SYNDROIME WITH CASTLEMAN DISEASE - AN UNUSUAL PRESENTATION
}

KEY WORDS:

Dr Gaurav Shah*

\section{Dr Shrinivas}

Andelwar

Dr Pranav Joshi

Dr Shalin Shah

Dr Mayank Patel

Dr Sudhir Shah
Resident, Department of Neurology, Smt. NHLMMC. Ahmedabad. *Corresponding Author

Resident, Department of Neurology, Smt. NHLMMC.Ahmedabad.

Assistant Professor, Department of Neurology, Smt.NHLMMC.Ahmedabad.

Assistant Professor, Department of Neurology, Smt.NHLMMC.Ahmedabad.

Associate Professor, Department of Neurology, Smt.NHLMMC. Ahmedabad.

Professor and HOD, Department of Neurology, Smt.NHLMMC. Ahmedabad.

Chronic Inflammatory Demyelinating Polyneuropathy(CIDP) is an acquired immune mediated disorder presenting as symmetric proximal and distal sensory motor polyneuropathy. It is treatable with immunosuppressant. CIDP may be associated with HIV-l infection, SLE, para neoplastic disorders and Paraproteinemias. Paraproteinemias is abnormal monoclonal antibody or light chain in blood secondary to plasma cell disorder like multiple myeloma, Waldenstrom macroglobulinemia, monoclonal gammopathy of undetermined significance (MUGS), POEMS (Polyneuropathy, Organomegaly, Endocrinopathy, monoclonal plasma cell proliferative disorder and Skin Changes) syndrome and Castleman disease with or without POEMS syndrome. These account for approximately 10\% of CIDP. Diagnosing Paraproteinemic neuropathy is necessary to prevent delay in diagnosis, start appropriate treatment and estimate prognosis of the condition.

POEMS syndrome is due to clonal proliferation of plasma cells. Castleman disease (CD), also known as angiofollicular or giant lymph node hyperplasia, is a rare non- neoplastic lymphoproliferative disorder.

We present a Case report of relatively young male with right parotid swelling with chronic sensorimotor quadriparesis with wasting, with no predominant neuropathic pain and cranial nerve involvement. On Nerve Conduction Studies it was found to have severe sensori-motor neuropathy which was not associated with pain, an unlikely feature of either CIDP or Castleman disease; on further evaluation was found to have POEMS syndrome. Thus high index of suspicion is necessary to diagnose this condition.

This case report emphasizes the fact that patients with Castleman disease-POEMS Syndrome may present at younger age than usual with short duration of illness, with severe sensorimotor neuropathy which may not be associated with pain. High index of suspicion and appropriate investigations may lead to early diagnosis and prompt treatment.

\section{INTRODUCTION:}

Polyneuropathy is one of the most common disorders of peripheral nervous system in adults with prevalence of around $5-8 \%$. Axonal neuropathy is more common than demyelinating polyneuropathy which is usually associated with Diabetes mellitus followed by chronic alcoholism. Among demyelinating polyneuropathy CIDP is one of the important differential to be considered. It may be associated with HIV-l infection, SLE, Chronic active hepatitis, Paraproteinemias and paraneoplastic disorders. Paraproteinemia is an abnormal monoclonal antibody or light chain in blood secondary to plasma cell disorder ranging from multiple myeloma, Waldenstrom macroglobulinemia, monoclonal gammopathy of undetermined significance (MUGS), to POEMS (Polyneuropathy, Organomegaly, Endocrinopathy, monoclonal plasma cell proliferative disorder and Skin Changes) syndrome. Neuropathy secondary to Paraproteinemias is one of the uncommon causes of chronic polyneuropathy that does not respond to conventional therapy.

\section{Case report:}

A 42 year old male on came to OPD with complains of right parotid swelling since 4 years, insidious onset gradually progressive chronic sensorimotor quadriparesis of all 4 limbs since 1 year started initially in the lower limb followed by upper limb, weakness was distal more than proximal. He also had paresthesia of all 4 limbs. He had no cranial nerve or bowel-bladder involvement.

On examination, patient was conscious and oriented; he needed two person support to stand and required assistance in activities of daily living. His general examination revealed
Hypertrichosis and Right parotid swelling of $10 * 8 \mathrm{~cm}$ which was hard and immobile. His Higher mental function and cranial nerve examination was normal. He had hypotonia and weakness with distal wasting of all 4 limbs (lower limbs> upper limbs, Distal $>$ proximal weakness), Power at shoulder joint was 4/5, and at Elbow, wrist, Hip, knee and ankle was 2/5. All Deep tendon reflexes were diminished and had impaired sensation in graded fashion over limbs. He had no Cerebellar or autonomic nervous system involvement. His Cardiovascular and respiratory system examination was normal, on abdominal examination hepatosplenomegaly was found.

His metabolic parameters were normal apart from ESR which was raised. His Thyroid function test showed hypothyroidism. Nerve conduction studies showed no elicitable response for any upper and lower limb motor or sensory nerves ,cerebrospinal fluid analysis showed- raised protein levels $(138 \mathrm{mg} / \mathrm{dl})$, while sugar $(78 \mathrm{mg} / \mathrm{dl})$ and cell count $(4$ cells-100\% lymphocytes) were normal. Autoimmune etiology workup (Serum ANA, Serum ANCA, Serum ACE levels) were negative.

Serum Immunoelectrophoresis was done to evaluate for paraproteins showed $M$ band with lambda chain suggestive of mono clonal gammopathy. To look for site of extravascular involvement by plasma cells CECT abdomen, thorax with lymph node screening was done which showed hepatomegaly, splenomegaly, and cervical lymphadenopathy. Bone marrow examination (PSIS) showed hypercellular marrow with myeloid hyperplasia and adequate megakaryocytes.

Upon further evaluation relatives found a report of Trephine 
Biopsy of Parotid mass in 2015 which showed focal plasmacytosis, suggestive of Castleman disease (Plasma cell type).He was treated with high dose IV steroids for 5 days without any significant improvement in weakness.

This patient had, severe sensorymotor polyneuropathy, with Monoclonal antibodies in blood, Endocrinopathy (hypothyroidism), Organomegaly (Hepato-splenomegaly), Skin changes (hypertrichosis) with biopsy proven Castleman disease, So, Castleman disease with POEMS syndrome (CDPOEMS) was considered. He was referred to oncologist for further management.

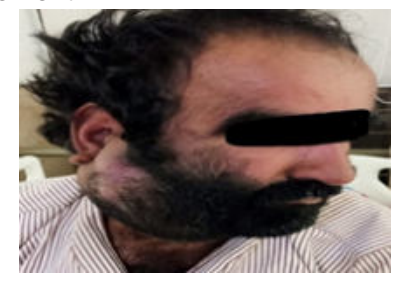

Fig 1: Patient has a hard immobile parotid swelling on right side.

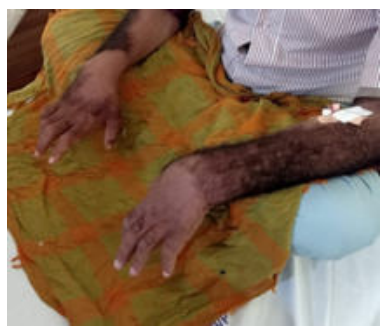

Fig 2: Patient has wasting with weakness of both the hands.

\section{DISCUSSION:}

The causes of Chronic Inflammatory Demyelinating Polyneuropathy (CIDP) are HIV-l infection, SLE, Paraneoplastic disorders, Chronic active hepatitis, Lymphomas, Irritable bowel disorder, Paraproteinemieas and Drugs. Paraproteinemias is abnormal monoclonal antibody or light chain in blood secondary to plasma cell disorder like multiple myeloma, Waldenstrom macroglobulinemia, monoclonal gammopathy of undetermined significance (MUGS), POEMS (Polyneuropathy, Organomegaly, Endocrinopathy, monoclonal plasma cell proliferative disorder and Skin Changes) syndrome and Castleman disease with or without POEMS syndrome. POEMS syndrome is due to clonal proliferation of plasma cells. Castleman disease (CD), also known as angiofollicular or giant lymph node hyperplasia, is a rare non- neoplastic lymphoproliferative disorder.

Castleman disease with POEMS syndrome(CD-POEMS) being a rare entity, little is known about its specific clinical and electro diagnostic criteria. It is more common in 6th decade with slightly male predominance, The mean duration of neuropathic symptoms is 48 months which are weakness(70\%), Numbness(95\%),Paresthesieas(85\%) and Neuropathic pain (55\%) as identified in case reports of Ge,Y. et al, ${ }^{6}$ Kang J., et al. ${ }^{7}$ and a case series of 20 patients by Naddaf et al., ${ }^{3}$. Patient in this case is younger than described and has neuropathic symptoms i.e numbness and weakness for approximately 12 months duration but was not associated with pain.The peripheral neuropathy in patients with Castleman disease tends to be more subtle than that of POEMS and is often sensory.

POEMS syndrome is clonal proliferation of plasma cells described by acronym Polyneuropathy, Organomegaly, Endocrinopathy, monoclonal plasma cell proliferative disorder and Skin Changes.It has prevalence of approximately $0.3 / 1,00,000$ population ${ }^{1}$.
Criteria for the diagnosis of POEMS syndrome ${ }^{1}$ include

Mandatory major criteria

1. Polyneuropathy (typically demyelinating)

2. Monoclonal plasma cell-proliferative disorder (almost always $\lambda$ )

Other major criteria (one required)

3. Castleman disease

4. Sclerotic bone lesions

5. Vascular endothelial growth factor elevation

Minor criteria

6. Organomegaly (splenomegaly, hepatomegaly, or lymphadenopathy)

7. Extravascular volume overload (edema, pleural effusion, or ascites)

8. Endocrinopathy (adrenal, thyroid, pituitary, gonadal, parathyroid, pancreatic)

9. Skin changes (hyperpigmentation, hypertrichosis, glomeruloid hemangiomata,plethora, acrocyanosis, flushing, white nails)

10. Papilledema

11. Thrombocytosis/polycythemia

Other symptoms and signs

Clubbing, weight loss, hyperhidrosis, pulmonary hypertension/ restrictive lung disease, thrombotic diatheses, diarrhea, low vitamin $\mathrm{B} 12$ values

The diagnosis of POEMS syndrome is confirmed when both of the mandatory major criteria, 1 of the 3 other major criteria, and 1 of the 6 minor criteria are present.Other names areTakatsuki syndrome, Crow-Fukase syndrome, osteosclerotic myeloma ${ }^{3}$.

The pathophysiologic mechanism causing neuropathy is not completely understood, but peripheral neuropathy is attributable to increase in inflammatory cytokines rather than clonal plasma cell invasion. VEGF along with other cytokines like TNF-alpha, IL-6, IL-12 play role in regulation of angiogenesis and microvascular permeability by targeting endothelial cell receptor. ${ }^{5}$

Endothelial injury occurs directly or indirectly secondary to abnormal activation of endothelial cells by VEGF. This leads to hypertrophy and proliferation of endothelial cells with secondary microangiopathy. This causes reduced oxygen supply, which in turn increases expression of Hypoxia inducible transcription factor -1(HIF-la) with secondary increase in VEGF.

The neuropathy presents sub-acutely in a length dependent manner with distal symmetric sensory symptoms followed by weakness.

Castleman disease is rare lymphoproliferative disorder characterized by enlarged lymph nodes- which may be Unicentric or Multicentric lymphadenopathy and a wide spectrum of clinical manifestations ${ }^{4}$. Peripheral neuropathy occurs in approximately $27 \%$ of patients with $\mathrm{CD}^{2}$. They usually present in sixth decade with lower limb numbness.

Compared to CIDP, Neuropathy with POEMS syndrome are rapidly disabling and painful, has prominent lower limb weakness and atrophy with lesser involvement of cranial nerve, and have some systemic features (endocrinopathy, organomegaly, skin changes among few), abnormal Paraproteins and increased levels of VEGF in blood. It is resistant to most of the standard treatment given in CIDP and have unpredictable prognosis. Castleman disease (CD) is seen in a subset of patients with POEMS syndrome, and when present, the neuropathy is similar to POEMS syndrome but is less severe. In Patients with purely $C D$, the neuropathy is often a mild, painless distal sensory neuropathy 
Many disorders with monoclonal gammopathy have demyelinating type of peripheral neuropathy, but it could not be characterized as patient had absent response on nerve conduction studies.Patients with Castleman disease and peripheral neuropathy are more likely to have monoclonal lambda protein in their serum and urine ${ }^{8}$.

Treatment of POEMS syndrome is directed at underlying monoclonal plasma cell disorder and extent of disease and bone marrow involvement. In patients with two or fewer plasmacytoma, radiotherapy is first line therapy ${ }^{3}$. Patients with generalized disease should be treated with systemic therapy. However high dose chemotherapy conditioned autologous stem cell transplantation is current gold standard treatment of POEMS syndrome. The Potential newer approaches involve targeting VEGF, IL-6,IL-2 and TNF.Agents like thalidomide, lenolidomide and bortezomib are candidates to treat patients with high risk of stem cell transplantation because of their effectiveness against plasma cell proliferation. They also have anti-VEGF and Anti-cytokine effect. $^{5}$

The median age of survival of patients with POEMS syndrome is 14 years, the presence of severe neuropathy in later stages of disease is associated with poor response with treatment and worse outcomes.

Thus,This case report emphasizes the fact that patients with Castleman disease-POEMS Syndrome may present at younger age than usual with short duration of illness, with severe sensorimotor neuropathy which may not be associated with pain. A High index of suspicion and appropriate investigations may lead to early diagnosis and prompt treatment.

\section{REFERENCES}

1. Dispenzieri, A. POEMS Syndrome :2019 Update on diagnosis, risk stratification, and management.AmJ Hematol.2019;94:812-827

2. Dispenzieri A, Armitage JO, Loe MJ, et al. The clinical spectrum of Castleman's disease.Am J Hematol2012;87(11):997-1002.

3. Naddaf E, Dispenzieri A, Mandrekar J, et al. Clinical spectrum of Castlemandisease-associated neuropathy. Neurology 2016;87(23):2457-62.

4. Mauermann, Michelle $L$, the peripheral neuropathies of POEMS syndrome and Castleman Disease. Hematology/Oncology Clinics, volume 32, issue 1, (2018)153-163

5. Cerri,F.,Falzone,Y.,Riva, N.,Quattrini,A.(2019).An update on the diagnosis and management of the polyneuropathy of POEMS Syndrome. Journal of Neurology, 266(1),258-267.

6. Ge,Y,Da,Q. and Dai,Y. Castleman disease of the hyaline vascular variant transforming into POEMS syndrome as end point:a case report. BMC Neurol 18,169(2018).

7. Kang J, Yang F, Zhang HY, Hu MM, Xia F, Wang JC, Deng YC , Zhao G. POEMS syndrome associated with Castleman disease: a case report and literature review.Neuroimmunol Neuroinflammation 2014;1:40-43.

8. Dispenzieri A, Moreno-Aspitia A, Lacy MQ, et al. Peripheral blood stem cell transplant (PBSCT) in a large series of patients with POEMS syndrome. Biol Blood Marrow Transplant.2004;10(2):14-15. 